\title{
Update on vaccination for the prevention of infectious respiratory disease in adults
}

\section{AtuAlizAÇÃo EM VACINAÇÃo NA PREVEnÇÃo dAS DOENÇAS RESPIRATÓRIAS INFECCIOSAS EM ADULTOS}

Wanderley M. Bernardo, Bernardo Maranhão, Fernando Lundgren, José Miguel Chatkin, Marcelo Fouad Rabahi, Nathalia Carvalho de Andrada, Ricardo Amorim Corrêa, Ricardo Martins, Roberto Stiburlov

1. Is there benefit in vaccine combination for the prevention of infectious respiratory diseases in adults?

a. Yes, because it reduces the cases of invasive pneumococcal disease (IPD) in the elderly.

b. Both the anti-influenza and pneumococcal vaccines reduce hospitalizations.

c. The association of a vaccine against Haemophilus influenzae B and a pneumococcal vaccine reduces by $5 \%$ the cases of IPD in healthy children.

d. There is no benefit in combining vaccines.

2. Are there any differences between pneumococcal polysaccharide vaccines (VPPS-23) and conjugate vaccines?

a. The VPPS-23 reduces the number of pneumonias and mortality.

b. Non-conjugate vaccines generate longer lasting and stronger immune response than conjugate vaccines.

c. The pneumococcal vaccine is not recommended for pregnant women.

d. There is no difference between VPPS-23 and conjugate vaccines.

3. Regarding the use of BCG vaccines, it is correct to say that:

a. The BCG vaccine is recommended for newborns through their first month of life.

b. The BCG vaccine is recommended for children up to two years old.

c. For adults, the only indication is related to tuberculosis in household contact.

d. In newborns, the recommended use is three doses of $0.1 \mathrm{~mL}$, every 3 months.

4. Are there benefits in using anti-pertussis vaccines?

a. The booster dose is not indicated because antibodies are high in individuals over 40 years old.

b. There are benefits in using the combined vaccine with inactivated polio. c. The immune response is better in adults between 65 and 74 years old.

d. Adults who live or work with infants or children under 1 year old should receive a single booster.

5. What are the indications for pneumococcal vaccine?

a. All adults.

b. Anyone between 2 and 64 years old who has a chronic disease.

c. Only adults aged 64 to 75 years, smokers or patients with asthma.

d. It is contraindicated for patients with immunosuppressive disease.

\section{Answers to clinical scenario: update on chronic nonspecific lower back pain: rehabilitation [published in RAMB 2014; 60]}

1. What is the benefit of acupuncture in the treatment of chronic nonspecific lower back pain? Acupuncture is less effective than massage. (alternative $\mathbf{D}$ )

2. What are the advantages of the combination of analgesics and muscle relaxant?

Carisoprodol has a potential for psychological and physical dependence. (alternative B)

\section{Which resources are used?}

The use of shortwave diathermy is recommended. (alternative $\mathbf{A}$ )

4. What is the role of electrical stimulation in chronic nonspecific lower back pain?

TENS has the advantage to be taken at home. (alternative $\mathbf{D}$ )

5. Is the use of antidepressants effective for treating nonspecific lower back pain?

Combined administration of antidepressants with adrenergic effects produces better results than the use of selective serotonin reuptake inhibitors. (alternative $\mathbf{A}$ ) 